\title{
Beyond mean-field approach to heavy-ion reactions around the Coulomb barrier
}

\author{
Kouhei Washiyama ${ }^{1, a}$, Denis Lacroix ${ }^{2}$, Sakir Ayik $^{3}$, and Bülent Yilmaz ${ }^{4}$ \\ 1 PNTPM, CP229, Université Libre de Bruxelles, 1050 Bruxelles, Belgium \\ 2 GANIL, BP55027, 14076 Caen, France \\ 3 Physics Department, Tennessee Technological University, Cookeville, Tennessee 38505, USA \\ 4 Physics Department, Ankara University, Tandogan 06100, Ankara, Turkey
}

\begin{abstract}
Dissipation and fluctuations of one-body observables in heavy-ion reactions around the Coulomb barrier are investigated with a microscopic stochastic mean-field approach. By projecting the stochastic meanfield dynamics on a suitable collective path, transport coefficients associated with the relative distance between colliding nuclei and a fragment mass are extracted. Although microscopic mean-field approach is know to underestimate the variance of fragment mass distribution, the description of the variance is much improved by the stochastic mean-field method. While fluctuations are consistent with the empirical (semiclassical) analysis of the experimental data, concerning mean values of macroscopic variables the semiclassical description breaks down below the Coulomb barrier.
\end{abstract}

\section{Introduction}

The interplay between nuclear structure and dynamical effects is crucial to properly describing heavy-ion fusion reactions at energies close to the Coulomb barrier. The meanfield theory based on the Skyrme energy density functional provides a rather unique tool for describing nuclear structure and nuclear reactions over the whole nuclear chart in a unified framework. For nuclear dynamical calculations, time-dependent Hartree-Fock (TDHF) model has been developed [1-4]. This model automatically includes important dynamical effects such as vibration of nuclei, neck formation, and nucleon transfer during reactions. Most recent TDHF simulations are able to include all terms of the Skyrme energy density functional, for example the spinorbit terms, and to break spacial symmetries [5-10]. To perform full three-dimensional calculations and to use effective forces consistent with static calculations is crucial to accounting for the richness of nuclear shapes in dynamical evolution.

In the mean-field theory, short range two-body correlations are neglected and nucleons move in the self-consistent potential produced by all other nucleons. This is a good approximation at low energies since Pauli blocking is effective for scattering into unoccupied states. Consequently, collective energy is converted into intrinsic degrees of freedom via interaction of nucleons with the self-consistent mean field, so-called one-body dissipation. One-body dissipation mechanism plays a dominant role in low energy nuclear dynamics. One important limitation of the meanfield theory is related with dynamical fluctuations of collective variables. In the mean-field description, while single-

\footnotetext{
a e-mail: kouhei.washiyama@ulb.ac.be
}

particle motion is treated in a quantal framework, collective motion is treated almost in a classical approximation. Therefore, TDHF provides a good description for average evolution; however it severely underestimates fluctuations of collective variables [11-13].

After the first application of the TDHF theory, much effort has been devoted to overcoming this difficulty and to developing transport theories that are able to describe not only mean values but also fluctuations (for a review, see Refs $[14,15])$. Among them, the variational principle by Balian and Vénéroni appears as one of the most promising methods $[16,17]$. However, even nowadays it remains difficult to apply $[18,19]$. The absence of a practical solution to include fluctuations beyond mean field in a fully microscopic framework strongly restricts applications of mean-field-based theories to low energy nuclear reactions.

A stochastic mean-field (SMF) approach has been proposed for describing fluctuation dynamics [20]. For small amplitude fluctuations, this model gives a result for dispersion of a one-body observable that is identical to the result obtained through a variational approach [16].

In this contribution, first, we discuss the property of nucleus-nucleus potential and one-body dissipation deduced from TDHF calculations. They are not constrained by adiabatic or diabatic approximation. Therefore, this method should provide an accurate description of nucleus-nucleus potential and one-body dissipation at energies around the Coulomb barrier [21,22]. Then, we discuss how to overcome the failure of description of fluctuations of one-body observables in TDHF and employ the SMF approach [20, 23]. We project the SMF evolution on a collective path to obtain the expression of diffusion coefficients. We show that the description of the variance of fragment mass dis- 
tributions in transfer reactions by the stochastic mean-field approach is much improved compared to that of the TDHF model [24].

\section{Nucleus-nucleus potential and one-body dissipation from mean-field dynamics}

Nucleus-nucleus potential and one-body dissipation are extracted as follows [21,22]: (i) We solve the TDHF equation for head-on collisions to obtain the time evolution of the total density of colliding nuclei. (ii) Defining a separation plane between two nuclei, we compute at each time the relative distance $R$, associated momentum $P$, and reduced mass $\mu$ of colliding nuclei. (iii) We assume that mean-field evolution obeys a classical equation of motion with a friction term:

$$
\frac{d R}{d t}=\frac{P}{\mu}, \quad \frac{d P}{d t}=-\frac{d V}{d R}-\gamma(R) \dot{R},
$$

where $V(R)$ and $\gamma(R)$ denote the nucleus-nucleus potential and friction coefficient, respectively. The friction coefficient $\gamma(R)$ describes the effect of one-body energy dissipation from the macroscopic collective degrees of freedom to the microscopic ones. For the TDHF calculations presented in this contribution, the three-dimensional TDHF code developed by P. Bonche and coworkers with the SLy4d Skyrme effective force [5] is used. The mesh sizes in space and in time are $0.8 \mathrm{fm}$ and $0.45 \mathrm{fm} / c$, respectively. For more details, see Refs. [21,22].

Figure 1 shows the difference between the barrier height deduced from TDHF evolution $\left(V_{B}\right)$ and the experimental barrier height $\left(V_{B}^{\exp }\right)$ [25] as a function of $V_{B}$. The solid line corresponds to the barrier height extracted using highenergy TDHF trajectories $\left(E_{\mathrm{c} . \mathrm{m} .} \gg V_{B}\right)$, whereas the dashed line is the result for low-energy TDHF trajectories $\left(E_{\mathrm{c} . \mathrm{m} .} \sim\right.$ $\left.V_{B}\right)$. The former identifies with the barrier height of the frozen density approximation [26]. Dynamical reduction of the barrier height from high-energy TDHF to low-energy TDHF is clearly seen for all reactions. Moreover, because of this reduction, the value of the barrier height at low energy approaches the experimental data. This underlines the importance of dynamical effects close to the Coulomb barrier and shows the precision of our method.

This method also provides information on one-body dissipation through the friction coefficient $\gamma$. In Fig. 2, we present reduced friction coefficients $\beta(R) \equiv \gamma(R) / \mu$ as a function of $R$ scaled by the Coulomb barrier radius $R_{B}$ for different systems. This clearly shows that the order of magnitude of $\beta(R)$ and the radial dependence are almost independent on the size and asymmetry of the system. We compare these friction coefficients with that of a microscopic model based on small amplitude response by Adamian et al. [27] by the solid circles. The radial dependence and the magnitude of their friction coefficient are very similar to those extracted from TDHF evolution.

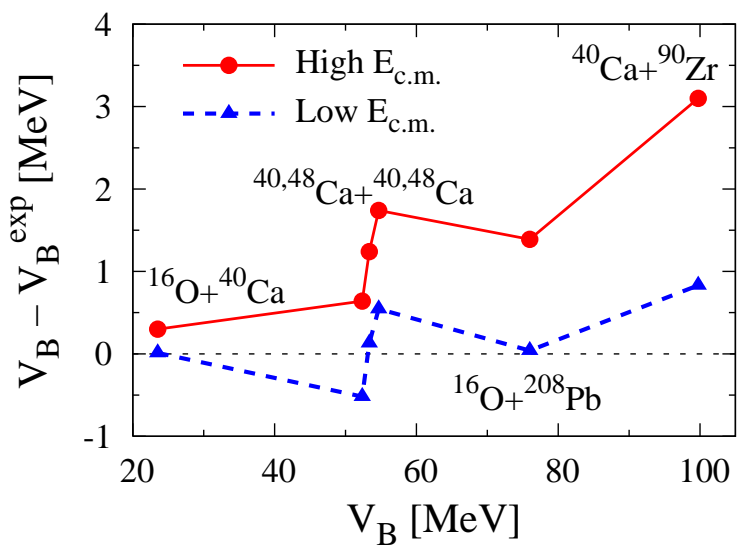

Fig. 1. Barrier height $V_{B}$ deduced from TDHF minus experimental barrier height $V_{B}^{\exp }$ as a function of extracted barrier height for the reactions indicated in the figure. The values $V_{B}$ are deduced from high energy (solid line) and from low energy $\left(E_{\text {c.m. }} \sim V_{B}\right)$ (dashed line) TDHF trajectories, respectively.

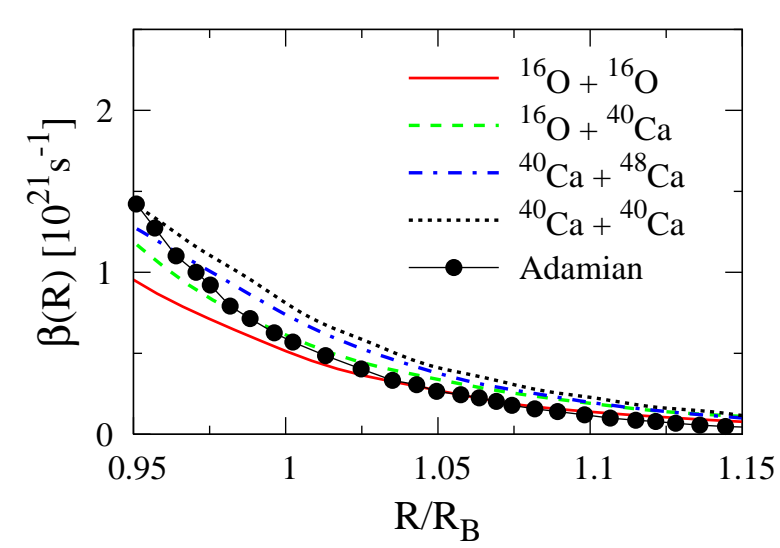

Fig. 2. Extracted reduced friction $\beta(R) \equiv \gamma(R) / \mu$ as a function of $R$ scaled by the Coulomb barrier radius $R_{B}$ for different systems. A microscopic friction coefficient by Adamian et al. [27] is shown by the solid circles for comparison.

\section{Fluctuations of one-body observables from stochastic mean-field dynamics}

In the previous section, we show that mean-field dynamics gives a good description for the nucleus-nucleus potential and one-body dissipation. However, it is well known that TDHF can not reproduce fluctuations of one-body observables, for example the variance of fragment mass distributions in deep inelastic collisions [13], although TDHF calculations well reproduce the mean value of fragment mass.

In order to overcome this difficulty, we have recently proposed a stochastic mean-field (SMF) approach, which is a stochastic extension of the mean-field model for low energy nuclear dynamics so as to include zero-point fluctuations of the initial state $[20,23]$. The initial density fluctuations are simulated by representing the initial state in terms of a suitable ensemble of initial single-particle density matrices, which is similar to the idea in Refs. [28,29]. 
In this manner, the description with a single Slater determinant is replaced by a superposition of several Slater determinants. A member of the ensemble of density matrices, indicated by event label $\lambda$, can be expressed as

$$
\rho^{\lambda}\left(\mathbf{r}, \mathbf{r}^{\prime}, t\right)=\sum_{i j \sigma \tau} \Phi_{i \sigma \tau}^{*}(\mathbf{r}, t ; \lambda) \rho_{i j}^{\lambda}(\sigma \tau) \Phi_{j \sigma \tau}\left(\mathbf{r}^{\prime}, t ; \lambda\right),
$$

where the sums $i$ and $j$ run over a complete set of singleparticle wave functions $\Phi_{i \sigma \tau}(\mathbf{r}, t ; \lambda)$ with spin-isospin quantum numbers $\sigma, \tau$. According to the description of the SMF approach [20], matrix elements $\rho_{i j}^{\lambda}(\sigma \tau)$ are assumed to be time-independent random Gaussian numbers with a mean value $\overline{\rho_{i j}^{\lambda}(\sigma \tau)}=\delta_{i j} n_{i}^{\sigma \tau}$ and with a variance

$$
\begin{aligned}
& \overline{\delta \rho_{i j}^{\lambda}(\sigma \tau) \delta \rho_{j^{\prime} i^{\prime}}^{\lambda}\left(\sigma^{\prime} \tau^{\prime}\right)} \\
= & \frac{1}{2} \delta_{j j^{\prime}} \delta_{i i^{\prime}} \delta_{\tau \tau^{\prime}} \delta_{\sigma \sigma^{\prime}}\left[n_{i}^{\sigma \tau}\left(1-n_{j}^{\sigma \tau}\right)+n_{j}^{\sigma \tau}\left(1-n_{i}^{\sigma \tau}\right)\right],
\end{aligned}
$$

where $\bar{X}$ denotes the ensemble average of $X$. Here, $n_{i}^{\sigma \tau}$ denotes the average single-particle occupation factor. At zero temperature occupation factors are 0 and 1 , and at finite temperature they are determined by the Fermi-Dirac distribution. The great advantage of the SMF approach is that each Slater determinant evolves independently from each other following the time evolution of its single-particle wave functions in its self-consistent mean-field Hamiltonian, denoted by $h\left(\rho^{\lambda}\right)$, according to

$$
i \hbar \frac{\partial}{\partial t} \Phi_{i \sigma \tau}(\mathbf{r}, t ; \lambda)=h\left(\rho^{\lambda}\right) \Phi_{i \sigma \tau}(\mathbf{r}, t ; \lambda) .
$$

In the following applications, we focus on the headon collision along the $x$ axis around the Coulomb barrier energy.

\subsection{Fusion reactions}

First, we apply the SMF approach to fusion reactions [23]. We project the SMF evolution on a one-dimensional macroscopic Langevin equation, which is similar to Eq. (1) except an additional Gaussian random force $\xi_{P}^{\lambda}(t)$ :

$$
\frac{d}{d t} P^{\lambda}=-\frac{d}{d R^{\lambda}} U\left(R^{\lambda}\right)-\gamma\left(R^{\lambda}\right) \dot{R}^{\lambda}+\xi_{P}^{\lambda}(t),
$$

Ignoring non-Markovian effects, the random force $\xi_{p}^{\lambda}(t)$ with zero mean value reduces to white noise specified by the following correlation function:

$$
\overline{\xi_{P}^{\lambda}(t) \xi_{P}^{\lambda}\left(t^{\prime}\right)}=2 \delta\left(t-t^{\prime}\right) D_{P P}(R)
$$

Here $D_{P P}(R)$ denotes the momentum diffusion coefficient. Denoting $x_{0}$ as the position of the separation plane between the two nuclei ( $x_{0}=0$ in this case), we have the following semiclassical expression for the nucleon diffusion coefficient according to Ref. [23]:

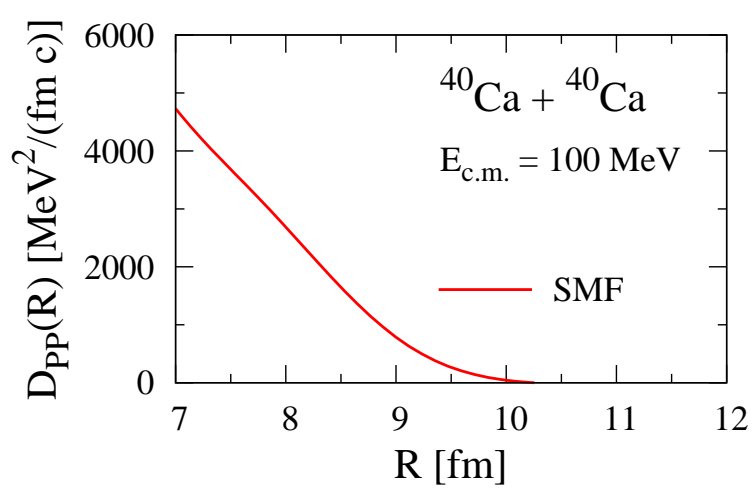

Fig. 3. Diffusion coefficient as a function of the relative distance for the head-on ${ }^{40} \mathrm{Ca}+{ }^{40} \mathrm{Ca}$ collision at $E_{\text {c.m. }}=100 \mathrm{MeV}$.

$$
\begin{aligned}
D_{P P}(t)= & \int \frac{d p_{x}}{2 \pi \hbar} \frac{\left|p_{x}\right|}{m} \frac{p_{x}^{2}}{2} \\
& \times \sum_{\sigma \tau}\left\{f_{P}^{\sigma \tau}\left(x_{0}, p_{x}, t\right)\left[1-f_{T}^{\sigma \tau}\left(x_{0}, p_{x}, t\right) / \Omega\left(x_{0}, t\right)\right]\right. \\
& \left.+f_{T}^{\sigma \tau}\left(x_{0}, p_{x}, t\right)\left[1-f_{P}^{\sigma \tau}\left(x_{0}, p_{x}, t\right)\right] / \Omega\left(x_{0}, t\right)\right\} .(7)
\end{aligned}
$$

Here $m$ is the nucleon mass, $\Omega\left(x_{0}, t\right)$ is the phase-space volume over the window given in Ref. [23], and

$$
\begin{aligned}
& f_{P / T}^{\sigma \tau}\left(x_{0}, p_{x}, t\right) \\
= & \iint d y d z \int d s_{x} \exp \left(-\frac{i}{\hbar} p_{x} s_{x}\right) \\
& \times \sum_{i \in P / T} \Phi_{i \sigma \tau}^{*}\left(x+\frac{s_{x}}{2}, y, z, t\right) n_{i}^{\sigma \tau} \Phi_{i \sigma \tau}\left(x-\frac{s_{x}}{2}, y, z, t\right)
\end{aligned}
$$

is the average value of the reduced Wigner distribution associated with single-particle wave functions originating from the projectile/target. We note that the expression of the diffusion coefficient (7) has the same form as that given by the phenomenological nucleon exchange model in Ref. [30]. We also note that diffusion coefficients can be evaluated in terms of the average TDHF evolution through the Wigner transformation (8).

As an example, the momentum diffusion coefficient for the head-on ${ }^{40} \mathrm{Ca}+{ }^{40} \mathrm{Ca}$ collision at $E_{\text {c.m. }}=100 \mathrm{MeV}$ is shown in Fig. 3.

\subsection{Variance of fragment mass distribution in transfer reactions}

We show another application of SMF to the variance of fragment mass distribution in transfer reactions to improve the mean-field description [24]. To do so, we investigate head-on transfer reactions at energies just below the Coulomb barrier, where nucleon exchange will occur during reaction, and estimate the variance of fragment mass distribution.

By projecting the SMF evolution on the collective space for the fragment mass number, time evolution of the mass 
number of the projectile-like fragment $A_{P}^{\lambda}$ for an event $\lambda$ is described by a Langevin equation [31],

$$
\frac{d}{d t} A_{P}^{\lambda}=v\left(A_{P}^{\lambda}, t\right)+\xi_{A}^{\lambda}(t)
$$

where $v\left(A_{P}^{\lambda}, t\right)$ denotes the drift term for nucleon transfer. The Gaussian white noise random force $\xi_{A}^{\lambda}(t)$ is determined with zero mean value and a correlation function,

$$
\overline{\xi_{A}^{\lambda}(t) \xi_{A}^{\lambda}\left(t^{\prime}\right)}=2 \delta\left(t-t^{\prime}\right) D_{A A},
$$

where $D_{A A}$ is the diffusion coefficient associated with nucleon exchange. The variance $\sigma_{A A}^{2}$ of fragment mass distribution is determined by small fluctuations of the mass number $\delta A_{P}^{\lambda}$ through $\sigma_{A A}^{2}(t)=\overline{\delta A_{P}^{\lambda} \delta A_{P}^{\lambda}}$. The expression of the diffusion coefficient $D_{A A}$ is similar to the momentum diffusion coefficient $D_{P P}$ except that $p_{x}^{2}$ is removed from the integral in Eq. (7). Again, we note that the nucleon diffusion coefficient can also be evaluated from the average TDHF evolution.

According to the Langevin equation, neglecting contributions from the drift term, the variance is related to the diffusion coefficient according to [31,32]

$$
\sigma_{A A}^{2}(t) \simeq 2 \int_{0}^{t} D_{A A}(s) d s .
$$

In the phenomenological nucleon exchange model, the relation $\sigma_{A A}^{2}(t)=N_{\text {exc }}(t)$ was obtained, where $N_{\text {exc }}(t)$ denotes the accumulated total number of exchanged nucleons until time $t$, and was extensively used to analyze the experimental data of mass variance $[33,34]$. In the following, to check whether the SMF approach satisfies the above relation, we estimate the both quantities by the SMF approach.

We carry out calculations for the head-on ${ }^{40} \mathrm{Ca}+{ }^{40} \mathrm{Ca}$ reaction at energies just below the Coulomb barrier. Figure 4 illustrates the dependence of diffusion coefficients at different center-of-mass energies. The Coulomb barrier

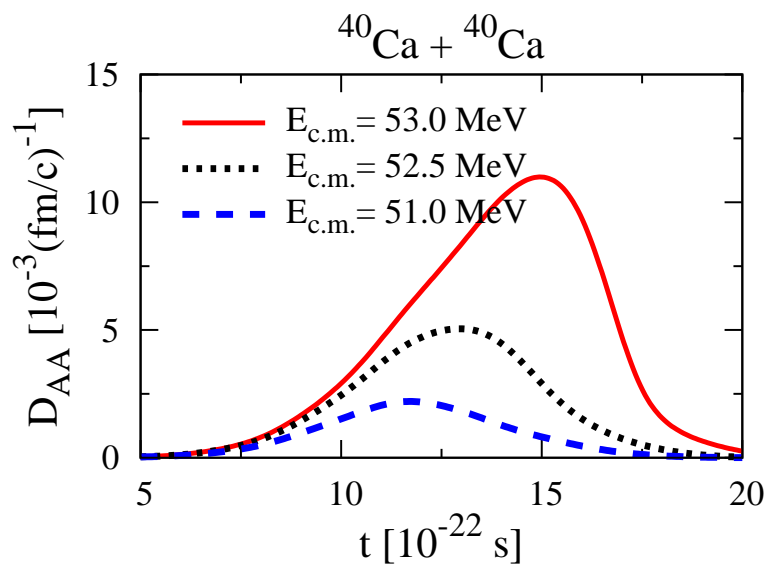

Fig. 4. Time evolution of diffusion coefficient calculated in the SMF approach for the head-on ${ }^{40} \mathrm{Ca}+{ }^{40} \mathrm{Ca}$ reaction at different center-of-mass energies below the Coulomb barrier.

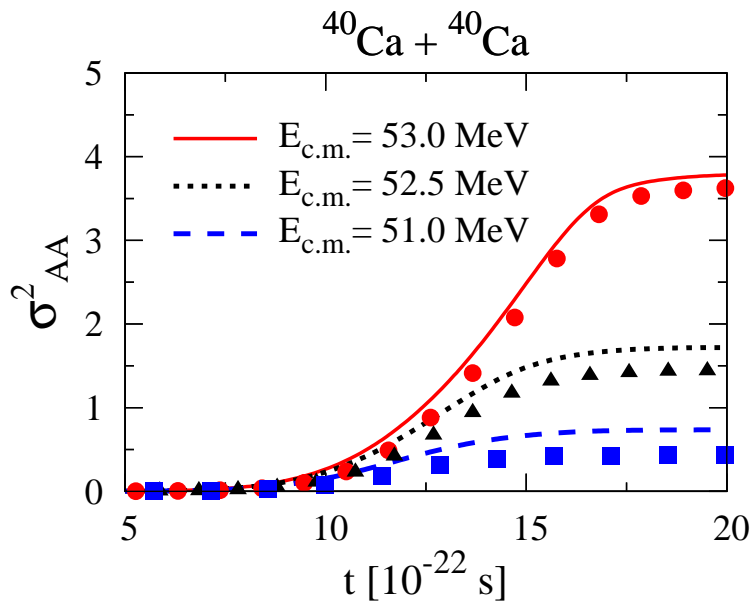

Fig. 5. Time evolution of $\sigma_{A A}^{2}$ obtained from the SMF approach for the ${ }^{40} \mathrm{Ca}+{ }^{40} \mathrm{Ca}$ reaction at the same energies as those in Fig. 4. Number of exchanged nucleon is superimposed by the solid circles, solid triangles, and solid squares from high to low energies, respectively.

energy of this system is $53.4 \mathrm{MeV}$. The magnitude of diffusion coefficient essentially depends on the size of the window, the larger the window the larger the rate of change of nucleon exchange [32]. At a given center-of-mass energy, the diffusion coefficient becomes maximum at the turning point where the size of the window is the largest. Also, because of increasing overlap of the projectile and the target, the magnitude of the diffusion coefficient increases with energy.

In Fig. 5, the variance of the fragment mass distributions deduced from the SMF approach at the same energies as those in Fig. 4 are shown by lines. The corresponding evolution of the number of exchanged nucleons is superimposed by the solid circles, solid triangles, and solid squares from high to low energies, respectively. The mass variance estimated from the SMF approach is consistent with this relation. We also estimate the variance of fragment mass distribution using the standard TDHF approach. In Table 1, the asymptotic values of the variances obtained from SMF and from TDHF are compared with those of the number of exchanged nucleons. The TDHF results are much smaller than the number of exchanged nucleons and are also much smaller than the results obtained from the SMF approach. The failure of the TDHF theory on the description of the

Table 1. Asymptotic values of the variances obtained from TDHF $\left(\sigma_{\mathrm{TDHF}}^{2}\right)$ and $\mathrm{SMF}\left(\sigma_{A A}^{2}\right)$ for the ${ }^{40} \mathrm{Ca}+{ }^{40} \mathrm{Ca}$ reaction. Asymptotic values of the number of exchanged nucleons are also given in the last column.

\begin{tabular}{llll}
\hline$E_{\text {c.m. }}$ & $\sigma_{\text {TDHF }}^{2}$ & $\sigma_{A A}^{2}$ & $N_{\text {exc }}$ \\
\hline 51.0 & 0.004 & 0.730 & 0.432 \\
52.5 & 0.004 & 1.718 & 1.441 \\
53.0 & 0.008 & 3.790 & 3.634
\end{tabular}


variance of the fragment mass distribution has been recognized for a long time as a major limitation of the mean-field theory [11-13]. It appears that the SMF approach cures this shortcoming of the mean-field theory. As seen from Fig. 5, not only the asymptotic value of $\sigma_{A A}^{2}$ but also the entire time evolution is very close to the evolution of $N_{\mathrm{exc}}(t)$.

\section{Nucleon drift in asymmetric system}

The work presented in the previous section has been extended to asymmetric systems. Then, in addition to the diffusion coefficient, the drift term in Eq. (9) connected to the average nucleon transfer is different from zero. Its technical details will be reported elsewhere [35]. In order to extend the SMF approach to the asymmetric system, including the motion of the neck position is necessary. Following the nucleon exchange picture [30], the drift term can be estimated through the semiclassical expression of the SMF approach:

$$
\begin{aligned}
v_{A}(t)= & \int \frac{d p_{x}}{2 \pi \hbar} \frac{\left|p_{x}-p_{0}\right|}{m} \\
& \times \sum_{\sigma \tau}\left[f_{P}^{\sigma \tau}\left(x_{0}, p_{x}, t\right)-f_{T}^{\sigma \tau}\left(x_{0}, p_{x}, t\right)\right]
\end{aligned}
$$

where $p_{0} / m$ is the velocity of the neck position. On the other hand, the drift term in TDHF is given with the help of the Wigner function as

$$
v_{A}(t)=-\int \frac{d p_{x}}{2 \pi \hbar} \frac{p_{x}-p_{0}}{m} f\left(x_{0}, p_{x}, t\right),
$$

where the Wigner function is given from Eq. (8) by

$$
f\left(x, p_{x}, t\right)=\sum_{\sigma \tau}\left[f_{P}^{\sigma \tau}\left(x, p_{x}, t\right)+f_{T}^{\sigma \tau}\left(x, p_{x}, t\right)\right] .
$$

The expression of Eq. (12) can be seen as a semiclassical version of Eq. (13). We have recently examined its validity by comparing the average number of nucleon transfer,

$$
A_{T}(t=\infty)-A_{T}(t=0)=\int_{0}^{\infty} d t v_{A}(t)
$$

where $A_{T}(t)$ denotes the mass number of the target-like fragment at time $t$.

Figure 6 shows the result of Eq. (15) obtained by TDHF and by the semiclassical expression of SMF as a function of center-of-mass energy for the head-on ${ }^{40} \mathrm{Ca}+{ }^{90} \mathrm{Zr}$ reaction. The semiclassical expression which has been used at energies above the Coulomb barrier leads to values consistent with its fully quantal counterpart. However, it clearly breaks down at energies below the Coulomb barrier. This is due to the fact that the effect of nucleon tunneling is not treated well in the semiclassical expression below the Coulomb barrier, underlying the necessity of quantal description of nucleon transfer.

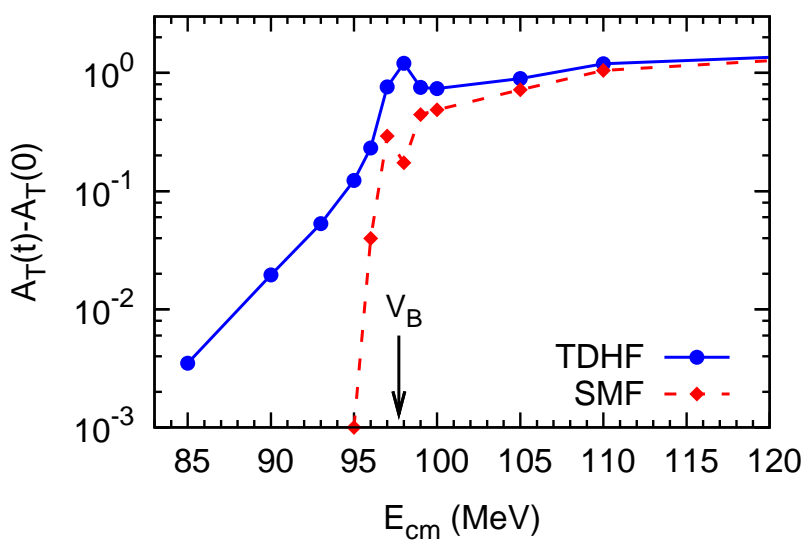

Fig. 6. The average number of nucleon transfer from ${ }^{40} \mathrm{Ca}$ to ${ }^{90} \mathrm{Zr}$ evaluated from TDHF (solid line) and from the semiclassical expression of SMF (dashed line) are shown as a function of centerof-mass energy for the head-on ${ }^{40} \mathrm{Ca}+{ }^{90} \mathrm{Zr}$ reaction. The Coulomb barrier $V_{B}$ is indicated as the arrow.

\section{Conclusion}

Mean-field dynamics and mean-field fluctuations using microscopic time-dependent models are discussed in the context of low energy nuclear reactions. We have shown that the TDHF theory gives precise values of nucleus-nucleus potential and a universal behavior of energy dissipation. By projecting the SMF equation on the relative distance of colliding nuclei and the mass number of the projectilelike nucleus, we extract the corresponding diffusion coefficients. We have shown that the SMF approach correctly describes the mass variance of final fragments in transfer reactions at energies near the Coulomb barrier. This gives a practical solution to properly describe mean-field fluctuations of one-body observables at low energies.

This work is supported in part by US DOE Grant DE-FG0589ER40530.

\section{References}

1. P. Bonche, S. E. Koonin, and J. W. Negele, Phys. Rev. C 13, (1976) 1226.

2. S. E. Koonin, Prog. Part. Nucl. Phys. 4, (1980) 283.

3. J. W. Negele, Rev. Mod. Phys. 54, (1982) 913.

4. C. Simenel, D. Lacroix, and B. Avez, Quantum ManyBody Dynamics: Applications to Nuclear Reactions (VDM Verlag, Sarrebruck, Germany, 2010).

5. K.-H. Kim, T. Otsuka, and P. Bonche, J. Phys. G 23, (1997) 1267.

6. C. Simenel, Ph. Chomaz, and G. de France, Phys. Rev. Lett. 86, (2001) 2971.

7. T. Nakatsukasa and K. Yabana, Phys. Rev. C 71, (2005) 024301.

8. J. A. Maruhn, P.-G. Reinhard, P. D. Stevenson, J. R. Stone, and M. R. Strayer, Phys. Rev. C 71, (2005) 064328. 
9. A. S. Umar and V. E. Oberacker, Phys. Rev. C 73, (2006) 054607.

10. Y. Iwata, T. Otsuka, J. A. Maruhn, and N. Itagaki, Phys. Rev. Lett. 104, (2010) 252501.

11. S. E. Koonin, K. T. R. Davies, B. Maruhn-Rezwani, H. Feldmeiner, S. J. Krieger, and J. W. Negele, Phys. Rev. C 15, (1977) 1359.

12. K. T. R. Davies, V. Maruhn-Rezwani, S. E. Koonin, and J. W. Negele, Phys. Rev. Lett. 41, (1978) 632.

13. C. H. Dasso, T. Døssing, and H. C. Pauli, Z. Phys. A 289, (1979) 395.

14. Y. Abe, S. Ayik, P.-G. Reinhard, and E. Suraud, Phys. Rep. 275, (1996) 49.

15. D. Lacroix, S. Ayik, and Ph. Chomaz, Prog. Part. Nucl. Phys. 52, (2004) 497.

16. R. Balian and M. Vénéroni, Phys. Lett. B136, (1984) 301; R. Balian, P. Bonche, H. Flocard, and M. Vénéroni, Nucl. Phys. A428, (1984) 79c.

17. J. B. Marston and S. E. Koonin, Phys. Rev. Lett. 54, (1985) 1139.

18. J. M. A. Broomfield and P. D. Stevenson, J. Phys. G 35, (2008) 095102.

19. C. Simenel, Phys. Rev. Lett. 106, (2011) 112502.

20. S. Ayik, Phys. Lett. B658, (2008) 174.

21. K. Washiyama and D. Lacroix, Phys. Rev. C 78, (2008) 024610.

22. K. Washiyama, D. Lacroix, and S. Ayik, Phys. Rev. C 79, (2009) 024609.

23. S. Ayik, K. Washiyama, and D. Lacroix, Phys. Rev. C 79, (2009) 054606.

24. K. Washiyama, S. Ayik, and D. Lacroix, Phys. Rev. C 80, (2009) 031602(R).

25. J. O. Newton et al., Phys. Lett. B586, (2004) 219; Phys. Rev. C 70, (2004) 024605.

26. V. Y. Denisov and W. Nörenberg, Eur. Phys. J. A 15, (2002) 375 .

27. G. G. Adamian, R. V. Jolos, A. K. Nasirov, and A. I. Muminov, Phys. Rev. C 56, (1997) 373.

28. H. Esbensen, A. Winther, R. A. Broglia, and C. H. Dasso, Phys. Rev. Lett. 41, (1978) 296.

29. C. H. Dasso and R. Donangelo, Phys. Lett. B276, (1992) 1.

30. H. Feldmeier, Rep. Prog. Phys. 50, (1987) 915.

31. J. Randrup and W. J. Swiatecki, Ann. Phys. (N.Y.) 125, (1980) 193; Nucl. Phys. A429, (1984) 105.

32. J. Randrup, Nucl. Phys. A383, (1982) 468.

33. H. Freiesleben and J. V. Kratz, Phys. Rep. 106, (1984) 1.

34. G. G. Adamian, A. K. Nasirov, N. V. Antonenko, and R. V. Jolos, Phys. Part. Nucl. 25, (1994) 583.

35. B. Yilmaz, S. Ayik, D. Lacroix, and K. Washiyama, in preparation. 